\title{
Relation between Quaternion Fourier Transform and Quaternion Wigner-Ville Distribution Associated with Linear Canonical Transform
}

\author{
Mawardi Bahri and Muh. Saleh Arif Fatimah \\ Department of Mathematics, Hasanuddin University, Makassar 90245, Indonesia \\ Correspondence should be addressed to Mawardi Bahri; mawardibahri@gmail.com
}

Received 16 April 2017; Accepted 27 July 2017; Published 27 September 2017

Academic Editor: Guowei Yang

Copyright (c) 2017 Mawardi Bahri and Muh. Saleh Arif Fatimah. This is an open access article distributed under the Creative Commons Attribution License, which permits unrestricted use, distribution, and reproduction in any medium, provided the original work is properly cited.

The quaternion Wigner-Ville distribution associated with linear canonical transform (QWVD-LCT) is a nontrivial generalization of the quaternion Wigner-Ville distribution to the linear canonical transform (LCT) domain. In the present paper, we establish a fundamental relationship between the QWVD-LCT and the quaternion Fourier transform (QFT). Based on this fact, we provide alternative proof of the well-known properties of the QWVD-LCT such as inversion formula and Moyal formula. We also discuss in detail the relationship among the QWVD-LCT and other generalized transforms. Finally, based on the basic relation between the quaternion ambiguity function associated with the linear canonical transform (QAF-LCT) and the QFT, we present some important properties of the QAF-LCT.

\section{Introduction}

The quaternion Fourier transform (QFT) is a nontrivial generalization of the real and complex classical Fourier transforms (FT) using quaternion algebra. Many useful properties of the QFT were obtained such as shift, modulation, convolution, correlation, differentiation, energy conservation, and uncertainty principle. It was first introduced in [1] for the analysis of $2 \mathrm{D}$ linear time-invariant partial differential systems and then applied in color image processing $[2,3]$. It is a natural question to extend the QFT to the linear canonical transform (LCT) domains and then it is the so-called quaternionic linear transform (QLCT). This extension is constructed by substituting the kernel of the QFT with the kernel of the LCT. A number of useful properties of the QLCT have been investigated including shift, orthogonality relation, reconstruction formula, and Heisenberg uncertainty principle (see, for example, [4-6] and the references given therein).

In [7], the author studied that the fractional Fourier transform (FrFT) can be reduced to the classical Fourier transform. Based on this fact, some properties of the FrFT can be derived very easily from those of the classical Fourier transform by simple change variable. Recently, in [8], the authors developed this idea to derive an uncertainty principle associated with the quaternion linear canonical transform (QLCT) by using the fundamental relationship between the QLCT and the QFT [8]. In [9], the authors proposed the quaternion Wigner-Ville distribution associated with linear canonical transform (QWVD-LCT) and obtain its fundamental properties. In the present paper, we first establish the basic relationship between the QWVD-LCT and the QFT. We then show that some fundamental properties of the QWVD-LCT such as inversion formula and Moyal formula can be obtained by combining this relation and the properties of the QFT. We investigate that the QWVD-LCT can be reduced to the quaternion windowed Fourier transform and the continuous quaternion Fourier transform. We finally establish the relationship between the QAF-LCT and the QFT which enables us to derive some useful properties of the QAFLCT.

\section{Preliminaries}

In the preliminaries we remind the reader of some facts of quaternions, the quaternion Fourier transform, and the quaternion linear canonical transform. 
2.1. Basic Facts about Quaternions. The quaternion algebra over $\mathbb{R}$, denoted by $\mathbb{M}$, is an associative noncommutative fourdimensional algebra:

$$
\mathbb{H}=\left\{q=q_{0}+\mathbf{i} q_{1}+\mathbf{j} q_{2}+\mathbf{k} q_{3} ; q_{0}, q_{1}, q_{2}, q_{3} \in \mathbb{R}\right\},
$$

which obeys the following multiplication rules:

$$
\begin{aligned}
& \mathbf{i j}=-\mathbf{j i}=\mathbf{k}, \\
& \mathbf{j k}=-\mathbf{k} \mathbf{j}=\mathbf{i}, \\
& \mathbf{k i}=-\mathbf{i k}=\mathbf{j}, \\
& \mathbf{i}^{2}=\mathbf{j}^{2}=\mathbf{k}^{2}=\mathbf{i j k}=-1 .
\end{aligned}
$$

For a quaternion $q=q_{0}+\mathbf{i} q_{1}+\mathbf{j} q_{2}+\mathbf{k} q_{3} \in \mathbb{H}, q_{0}$ is called the scalar part of $q$ denoted by $\operatorname{Sc}(q)$ and $\mathbf{i} q_{1}+\mathbf{j} q_{2}+\mathbf{k} q_{3}$ is called the vector (or pure) part of $q$. The vector part of $q$ is conventionally denoted by $\mathbf{q}$. Let $p, q \in \mathbb{H}$ and $\mathbf{p}, \mathbf{q}$ be their vector parts, respectively. Equation (2) yields the quaternionic multiplication $q p$ as

$$
q p=q_{0} p_{0}-\mathbf{q} \cdot \mathbf{p}+q_{0} \mathbf{p}+p_{0} \mathbf{q}+\mathbf{q} \times \mathbf{p}
$$

where $\mathbf{q} \cdot \mathbf{p}=\left(q_{1} p_{1}+q_{2} p_{2}+q_{3} p_{3}\right)$ and $\mathbf{q} \times \mathbf{p}=\mathbf{i}\left(q_{2} p_{3}-q_{3} p_{2}\right)+$ $\mathbf{j}\left(q_{3} p_{1}-q_{1} p_{3}\right)+\mathbf{k}\left(q_{1} p_{2}-q_{2} p_{1}\right)$.

The quaternion conjugate of $q$, given by

$$
\bar{q}=q_{0}-\mathbf{i} q_{1}-\mathbf{j} q_{2}-\mathbf{k} q_{3}, \quad q_{0}, q_{1}, q_{2}, q_{3} \in \mathbb{R},
$$

is an anti-involution; that is,

$$
\overline{q p}=\bar{p} \bar{q}
$$

From (4), we obtain the norm or modulus of $q \in \mathbb{M}$ defined as

$$
|q|=\sqrt{q \bar{q}}=\sqrt{q_{0}^{2}+q_{1}^{2}+q_{2}^{2}+q_{3}^{2}} .
$$

It is not difficult to see that

$$
|q p|=|q||p|, \quad \forall p, q \in \mathbb{H} .
$$

Furthermore, it is easily seen that

$$
|p q r|=|r q p|, \quad \forall p, q, q \in \mathbb{H} .
$$

Using conjugate (4) and the modulus of $q$, we can define the inverse of $q \in \mathbb{H} \backslash\{0\}$ as

$$
q^{-1}=\frac{\bar{q}}{|q|^{2}}
$$

which shows that $\mathbb{H}$ is a normed division algebra.

Now we observe that

$$
\operatorname{Sc}(p \bar{q})=\frac{1}{2}(p \bar{q}+q \bar{p})=q_{0} p_{0}+\mathbf{q} \cdot \mathbf{p} .
$$

This leads to the cyclic multiplication; that is,

$$
\mathrm{Sc}(p q r)=\mathrm{Sc}(r q p)=\mathrm{Sc}(q p r), \quad \forall p, q, r \in \mathbb{H} .
$$

We define an inner product for quaternion-valued functions $f, g: \mathbb{R}^{2} \rightarrow \mathbb{H}$ as follows:

$$
(f, g)=\int_{\mathbb{R}^{2}} f(\mathbf{x}) \overline{g(\mathbf{x})} d \mathbf{x}, \quad d \mathbf{x}=d x_{1} d x_{2}
$$

with symmetric real scalar part

$$
\langle f, g\rangle=\frac{1}{2}[(f, g)+(g, f)]=\mathrm{Sc} \int_{\mathbb{R}^{2}} f(\mathbf{x}) \bar{g}(\mathbf{x}) d \mathbf{x} .
$$

In particular, for $f=g$, we obtain the $L^{2}\left(\mathbb{R}^{2} ; \mathbb{U}\right)$-norm

$$
\|f\|=\sqrt{\langle f, f\rangle}=\left(\int_{\mathbb{R}^{2}}|f(\mathbf{x})|^{2} d \mathbf{x}\right)^{1 / 2} .
$$

This gives the $L^{p}\left(\mathbb{R}^{2} ; \mathbb{U}\right)$-norm

$$
\|f\|_{p}=\left(\int_{\mathbb{R}^{2}}|f(\mathbf{x})|^{p} d \mathbf{x}\right)^{1 / p}, \quad 1 \leq p<\infty .
$$

2.2. Quaternion Linear Canonical Transform and Its Basic Properties. In this section, we briefly discuss the definition of the two-sided QFT and the two-sided quaternion linear canonical transform (QLCT) (for simplicity of notation, we write the QFT and QLCT instead of the two-sided QFT and the two-sided QLCT, resp., in the next section). We further collect some basic properties of the QLCT, which will be very useful later on.

Definition 1. The QFT of $f \in L^{1}\left(\mathbb{R}^{2} ; \mathbb{H}\right)$ is the transform $\mathscr{F}_{q}\{f\}: \mathbb{R}^{2} \rightarrow \mathbb{U}$ given by the integral

$$
\mathscr{F}_{q}\{f\}(\boldsymbol{\omega})=\int_{\mathbb{R}^{2}} e^{-\mathbf{i} \omega_{1} x_{1}} f(\mathbf{x}) e^{-\mathbf{j} \omega_{2} x_{2}} d \mathbf{x},
$$

where $\mathbf{x}=x_{1} \mathbf{e}_{1}+x_{2} \mathbf{e}_{2}, \boldsymbol{\omega}=\omega_{1} \mathbf{e}_{1}+\omega_{2} \mathbf{e}_{2}$ and the quaternion exponential product $e^{-\mathbf{i} \omega_{1} x_{1}} e^{-\mathbf{j} \omega_{2} x_{2}}$ is the quaternion Fourier kernel. Here $\mathscr{F}_{q}$ is called the quaternion Fourier transform operator.

Definition 2. If $f \in L^{1}\left(\mathbb{R}^{2} ; \mathbb{M}\right)$ and $\mathscr{F}_{q}\{f\} \in L^{1}\left(\mathbb{R}^{2} ; \mathbb{U}\right)$, then the inverse transform of the QFT is given by

$$
\begin{aligned}
f(\mathbf{x}) & =\mathscr{F}_{q}^{-1}\left[\mathscr{F}_{q}\{f\}\right](\mathbf{x}) \\
& =\frac{1}{(2 \pi)^{2}} \int_{\mathbb{R}^{2}} e^{\mathbf{i} \omega_{1} x_{1}} \mathscr{F}_{q}\{f\}(\boldsymbol{\omega}) e^{\mathbf{j} \omega_{2} x_{2}} d \boldsymbol{\omega},
\end{aligned}
$$

where $\mathscr{F}_{q}^{-1}$ is called the inverse QFT operator.

A useful property of the QFT is stated in the following lemma, which is needed to derive Moyal formula of the quaternion Wigner-Ville distribution associated with the linear canonical transform (QWVD-LCT).

Lemma 3 (QFT Parseval). Let $f, g \in L^{1}\left(\mathbb{R}^{2} ; \mathbb{H}\right) \cap L^{2}\left(\mathbb{R}^{2} ; \mathbb{H}\right)$. The relation between $f, g$ and their $Q F T$ is given by

$$
\langle f, g\rangle=\left\langle\mathscr{F}_{q}\{f\}, \mathscr{F}_{q}\{g\}\right\rangle \text {. }
$$


In particular, with $f=g$, we get the QFT version of the Plancherel formula; that is,

$$
\|f\|^{2}=\left\|\mathscr{F}_{q}\{f\}\right\|^{2} .
$$

Definition 4 (QLCT definition). Suppose that $A_{1}=\left(a_{1}, b_{1}\right.$, $\left.c_{1}, d_{1}\right)$ and $A_{2}=\left(a_{2}, b_{2}, c_{2}, d_{2}\right)$ are real matrix parameters satisfying $\operatorname{det}\left(A_{1}\right)=\operatorname{det}\left(A_{2}\right)=1$. The QLCT of a quaternion signal $f \in L^{1}\left(\mathbb{R}^{2} ; \mathbb{U}\right)$ is defined by

$$
\begin{aligned}
& L_{A_{1}, A_{2}}^{\mathbb{\sharp}}\{f\}(\boldsymbol{\omega}) \\
& \quad=\int_{\mathbb{R}^{2}} K_{A_{1}}\left(x_{1}, \omega_{1}\right) f(\mathbf{x}) K_{A_{2}}\left(x_{2}, \omega_{2}\right) d \mathbf{x},
\end{aligned}
$$

where the kernel functions of the QLCT above are given by

$$
\begin{aligned}
& K_{A_{1}}\left(x_{1}, \omega_{1}\right) \\
& = \begin{cases}\frac{1}{\sqrt{2 \pi b_{1}}} e^{(\mathbf{i} / 2)\left(\left(a_{1} / b_{1}\right) x_{1}^{2}-\left(2 / b_{1}\right) x_{1} \omega_{1}+\left(d_{1} / b_{1}\right) \omega_{1}^{2}-\pi / 2\right)}, & \text { for } b_{1} \neq 0 \\
\sqrt{d_{1}} e^{\mathbf{i}\left(c_{1} d_{1} / 2\right) \omega_{1}^{2}}, & \text { for } b_{1}=0,\end{cases} \\
& K_{A_{2}}\left(x_{2}, \omega_{2}\right) \\
& = \begin{cases}\frac{1}{\sqrt{2 \pi b_{2}}} e^{(\mathbf{j} / 2)\left(\left(a_{2} / b_{2}\right) x_{2}^{2}-\left(2 / b_{2}\right) x_{2} \omega_{2}+\left(d_{2} / b_{2}\right) \omega_{2}^{2}-\pi / 2\right)}, & \text { for } b_{2} \neq 0 \\
\sqrt{d_{2}} e^{\mathbf{j}\left(c_{2} d_{2} / 2\right) \omega_{2}^{2}}, & \text { for } b_{2}=0 .\end{cases}
\end{aligned}
$$

From the definition of the QLCT, we can see easily that, when $b_{1} b_{2}=0$ and $b_{1}=b_{2}=0$, the QLCT of a signal is essentially a quaternion chirp multiplication. Therefore, in this work, we always assume that $b_{1} b_{2} \neq 0$.

Lemma 5. The QLCT of a signal $f$ can be seen as the QFT of the signal $f$ in the following form:

$$
\begin{aligned}
& L_{A_{1}, A_{2}}^{\mathbb{N}}\{f\}(\boldsymbol{\omega})=\frac{e^{-\mathbf{i}(\pi / 4)}}{\sqrt{2 \pi b_{1}}} \\
& \cdot e^{\left(\mathbf{i} d_{1} / 2 b_{1}\right) \omega_{1}^{2}} \mathscr{F}_{q}\left\{e^{\mathbf{i}\left(a_{1} / 2 b_{1}\right) x_{1}^{2}} f(\mathbf{x}) e^{\mathbf{j}\left(a_{2} / 2 b_{2}\right) x_{2}^{2}}\right\} \\
& \cdot\left(\frac{\omega_{1}}{b_{1}}, \frac{\omega_{2}}{b_{2}}\right) \frac{e^{-\mathbf{j}(\pi / 4)}}{\sqrt{2 \pi b_{2}}} e^{\left(\mathbf{j} d_{2} / 2 b_{2}\right) \omega_{2}^{2}} .
\end{aligned}
$$

It is worth noting that if $A_{1}=A_{2}=\left(a_{i}, b_{i}, c_{i}, d_{i}\right)=$ $(0,1,-1,0)$ for $i=1,2,(22)$ will reduce to the QFT definition; that is,

$$
\begin{aligned}
& L_{A_{1}, A_{2}}^{\mathbb{M}}\{f\}(\boldsymbol{\omega}) \\
& \quad=\int_{\mathbb{R}^{2}} \frac{e^{-\mathbf{i}(\pi / 4)}}{\sqrt{2 \pi}} e^{-\mathbf{i} \omega_{1} x_{1}} f(\mathbf{x}) e^{-\mathbf{j} \omega_{2} x_{2}} \frac{e^{-\mathbf{j}(\pi / 4)}}{\sqrt{2 \pi}} d \mathbf{x} \\
& \quad=\frac{e^{-\mathbf{i}(\pi / 4)}}{\sqrt{2 \pi}} \mathscr{F}_{q}\{f\}(\boldsymbol{\omega}) \frac{e^{-\mathbf{j}(\pi / 4)}}{\sqrt{2 \pi}} .
\end{aligned}
$$

Theorem 6 (QLCT Parseval). Two quaternion functions $f, h \in L^{1}\left(\mathbb{R}^{2} ; \mathbb{U}\right) \cap L^{2}\left(\mathbb{R}^{2} ; \mathbb{H}\right)$ are related to their $Q L C T$ via the Parseval formula, given as

$$
\langle f, h\rangle=\left\langle L_{A_{1}, A_{2}}^{\mathbb{N}}\{f\}, L_{A_{1}, A_{2}}^{\mathbb{Q}}\{h\}\right\rangle .
$$

When $f=h$, we get

$$
\|f\|^{2}=\left\|L_{A_{1}, A_{2}}^{\llbracket}\{f\}\right\|^{2}
$$

Proof. For a detailed proof of the above theorem, we refer the reader to [8].

\section{Quaternion Wigner-Ville Distribution and Quaternion Ambiguity Function in Linear Canonical Transform Domains}

Let us introduce the 2D quaternion Wigner-Ville distribution (QWVD) and quaternion ambiguity function QAF [10]. According to the QWVD and QAF definitions and the QLCT definition, we obtain a definition of the quaternion WignerVille distribution associated with the linear canonical transform (QWVD-LCT) and the quaternion ambiguity function associated with the linear canonical transform (QAF-LCT) (see [9]). We establish the fundamental relationship between the QWVD-LCT and QFT. Applying this relation and the properties of the QFT, we in detail derive the inverse transform formula and Moyal's formula for the QWVDLCT and the QAF-LCT, where the proof of the properties is quite different from one proposed in [9]. We also study the relationship among the QWVD-LCT, the quaternion windowed Fourier transform, and the continuous quaternion Fourier transform.

\subsection{Main Properties of QWVD-LCT and Relation among QAF- LCT, QWFT, and CQWT}

Definition 7. The cross quaternion Wigner-Ville distribution of two-dimensional functions (or signals) $f, g \in L^{2}\left(\mathbb{R}^{2} ; \mathbb{U}\right)$ is given by

$$
\begin{aligned}
& \mathscr{W}_{f, g}(\mathbf{x}, \boldsymbol{\omega}) \\
& \quad=\int_{\mathbb{R}^{2}} e^{-\mathbf{i} \omega_{1} \tau_{1}} f\left(\mathbf{x}+\frac{\boldsymbol{\tau}}{2}\right) \bar{g}\left(\mathbf{x}-\frac{\boldsymbol{\tau}}{2}\right) e^{-\mathbf{j} \omega_{2} \tau_{2}} d \boldsymbol{\tau},
\end{aligned}
$$

provided that the integral exists.

It should be remembered that the kernel of the cross QWVD in (26) does not commute with quaternion functions $f$ and $g$ so that several properties of the classical Wigner-Ville distribution (WVD) are not valid in the cross QWVD [10].

Definition 8. The cross quaternion ambiguity function of two-dimensional functions (or signals) $f, g \in L^{2}\left(\mathbb{R}^{2} ; \mathbb{H}\right)$ is given by

$$
\begin{aligned}
& \mathscr{A}_{f, g}(\mathbf{x}, \boldsymbol{\omega}) \\
& \quad=\int_{\mathbb{R}^{2}} e^{-\mathbf{i} \omega_{1} \tau_{1}} f\left(\boldsymbol{\tau}+\frac{\mathbf{x}}{2}\right) \bar{g}\left(\boldsymbol{\tau}-\frac{\mathbf{x}}{2}\right) e^{-\mathbf{j} \omega_{2} \tau_{2}} d \boldsymbol{\tau},
\end{aligned}
$$

provided that the integral exists. 
Definition 9. Suppose that $A_{1}=\left(a_{1}, b_{1}, c_{1}, d_{1}\right)$ and $A_{2}=\left(a_{2}\right.$, $\left.b_{2}, c_{2}, d_{2}\right)$ are real matrix parameters satisfying $\operatorname{det}\left(A_{1}\right)=$ $\operatorname{det}\left(A_{2}\right)=1$. The cross QWVD-LCT and cross QAF-LCT of a quaternion signal $f, g \in L^{1}\left(\mathbb{R}^{2} ; \mathbb{U}\right)$ are defined by

$$
\begin{aligned}
& \mathscr{W}_{f, \mathcal{g}}^{A_{1}, A_{2}}(\mathbf{x}, \boldsymbol{\omega})=\int_{\mathbb{R}^{2}} K_{A_{1}}\left(\tau_{1}, \omega_{1}\right) f\left(\mathbf{x}+\frac{\boldsymbol{\tau}}{2}\right) \\
& \cdot \bar{g}\left(\mathbf{x}-\frac{\boldsymbol{\tau}}{2}\right) K_{A_{2}}\left(\tau_{2}, \omega_{2}\right) d \boldsymbol{\tau} \\
& \mathscr{A}_{f, g}^{A_{1}, A_{2}}(\mathbf{x}, \boldsymbol{\omega})=\int_{\mathbb{R}^{2}} K_{A_{1}}\left(\tau_{1}, \omega_{1}\right) f\left(\boldsymbol{\tau}+\frac{\mathbf{x}}{2}\right) \\
& \cdot \bar{g}\left(\boldsymbol{\tau}-\frac{\mathbf{x}}{2}\right) K_{A_{2}}\left(\tau_{2}, \omega_{2}\right) d \boldsymbol{\tau} .
\end{aligned}
$$

Here the kernel functions of the above transforms are given by

$$
\begin{aligned}
& K_{A_{1}}\left(\tau_{1}, \omega_{1}\right) \\
& = \begin{cases}\frac{1}{\sqrt{2 \pi b_{1}}} e^{(\mathrm{i} / 2)\left(\left(a_{1} / b_{1}\right) \tau_{1}^{2}-\left(2 / b_{1}\right) \tau_{1} \omega_{1}+\left(d_{1} / b_{1}\right) \omega_{1}^{2}-\pi / 2\right)}, & \text { for } b_{1} \neq 0 \\
\sqrt{d_{1}} e^{\mathrm{i}\left(c_{1} d_{1} / 2\right) \omega_{1}^{2}}, & \text { for } b_{1}=0,\end{cases} \\
& K_{A_{2}}\left(\tau_{2}, \omega_{2}\right) \\
& = \begin{cases}\frac{1}{\sqrt{2 \pi b_{2}}} e^{(\mathrm{j} / 2)\left(\left(a_{2} / b_{2}\right) \tau_{2}^{2}-\left(2 / b_{2}\right) \tau_{2} \omega_{2}+\left(d_{2} / b_{2}\right) \omega_{2}^{2}-\pi / 2\right)}, & \text { for } b_{2} \neq 0 \\
\sqrt{d_{2}} e^{\mathrm{j}\left(c_{2} d_{2} / 2\right) \omega_{2}^{2}}, & \text { for } b_{2}=0 .\end{cases}
\end{aligned}
$$

It can be directly seen that if we write $h_{f, g}(\mathbf{x}, \boldsymbol{\tau})=f(\mathbf{x}+$ $\boldsymbol{\tau} / 2) \bar{g}(\mathbf{x}-\boldsymbol{\tau} / 2)$, we immediately obtain

$$
\begin{aligned}
& \mathscr{W}_{f, g}^{A_{1}, A_{2}}(\mathbf{x}, \boldsymbol{\omega}) \\
& =\int_{\mathbb{R}^{2}} \frac{1}{\sqrt{2 \pi b_{1}}} e^{(\mathbf{i} / 2)\left(\left(a_{1} / b_{1}\right) \tau_{1}^{2}-\left(2 / b_{1}\right) \tau_{1} \omega_{1}+\left(d_{1} / b_{1}\right) \omega_{1}^{2}-\pi / 2\right)} f(\mathbf{x} \\
& \left.+\frac{\boldsymbol{\tau}}{2}\right) \bar{g}\left(\mathbf{x}-\frac{\boldsymbol{\tau}}{2}\right) \\
& \quad \cdot \frac{1}{\sqrt{2 \pi b_{2}}} e^{(\mathrm{j} / 2)\left(\left(a_{2} / b_{2}\right) \tau_{2}^{2}-\left(2 / b_{2}\right) \tau_{2} \omega_{2}+\left(d_{2} / b_{2}\right) \omega_{2}^{2}-\pi / 2\right)} d \boldsymbol{\tau} \\
& =\int_{\mathbb{R}^{2}} \frac{1}{\sqrt{2 \pi b_{1}}} e^{(\mathbf{i} / 2)\left(\left(a_{1} / b_{1}\right) \tau_{1}^{2}-\left(2 / b_{1}\right) \tau_{1} \omega_{1}+\left(d_{1} / b_{1}\right) \omega_{1}^{2}-\pi / 2\right)} h_{f, g}(\mathbf{x}, \\
& \quad \boldsymbol{\tau}) \frac{1}{\sqrt{2 \pi b_{2}}} e^{(\mathbf{j} / 2)\left(\left(a_{2} / b_{2}\right) \tau_{2}^{2}-\left(2 / b_{2}\right) \tau_{2} \omega_{2}+\left(d_{2} / b_{2}\right) \omega_{2}^{2}-\pi / 2\right)} d \boldsymbol{\tau} \\
& =L_{A_{1}, A_{2}}^{\boxplus \Perp}\left\{h_{f, g}\right\}(\boldsymbol{\omega}) .
\end{aligned}
$$

This tells us that the cross QWVD-LCT is in fact the QLCT of the function $h_{f, g}(\mathbf{x}, \boldsymbol{\tau})$ with respect to $\tau$. This fact is very important in proving Moyal's formula for the cross QWVDLCT. Similarly, we also get

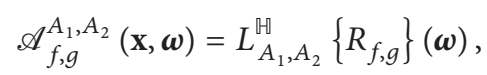

where $R_{f, g}$ is given by

$$
R_{f, g}(\mathbf{x}, \boldsymbol{\tau})=f\left(\boldsymbol{\tau}+\frac{\mathbf{x}}{2}\right) \bar{g}\left(\boldsymbol{\tau}-\frac{\mathbf{x}}{2}\right) .
$$

The following result presents an inequality related to the cross QWVD-LCT.

Lemma 10. Suppose that $f \in L^{p}\left(\mathbb{R}^{2} ; \mathbb{U}\right), g \in L^{q}\left(\mathbb{R}^{2} ; \mathbb{U}\right)$ with $1 / p+1 / q=1$. Then we have

$$
\left|\mathscr{W}_{f, g}^{A_{1}, A_{2}}(\mathbf{x}, \boldsymbol{\omega})\right| \leq \frac{1}{2 \pi \sqrt{b_{1} b_{2}}}\|f\|_{p}\|g\|_{q} .
$$

Proof. We straightforwardly obtain from (28) that

$$
\begin{aligned}
& \left|\mathscr{W}_{f, g}^{A_{1}, A_{2}}(\mathbf{x}, \boldsymbol{\omega})\right| \\
& =\mid \int_{\mathbb{R}^{2}} \frac{1}{\sqrt{2 \pi b_{1}}} e^{(\mathbf{i} / 2))\left(\left(a_{1} / b_{1}\right) \tau_{1}^{2}-\left(2 / b_{1}\right) \tau_{1} \omega_{1}+\left(d_{1} / b_{1}\right) \omega_{1}^{2}-\pi / 2\right)} f(\mathbf{x} \\
& \left.\quad+\frac{\boldsymbol{\tau}}{2}\right) \bar{g}\left(\mathbf{x}-\frac{\boldsymbol{\tau}}{2}\right) \times \frac{1}{\sqrt{2 \pi b_{2}}} \\
& \quad \cdot e^{(\mathbf{j} / 2)\left(\left(a_{2} / b_{2}\right) \tau_{2}^{2}-\left(2 / b_{2}\right) \tau_{2} \omega_{2}+\left(d_{2} / b_{2}\right) \omega_{2}^{2}-\pi / 2\right)} d \boldsymbol{\tau} \mid \leq\left(\int_{\mathbb{R}^{2}} \mid \frac{1}{\sqrt{2 \pi b_{1}}}\right. \\
& \left.\left.\quad \cdot e^{(\mathbf{i} / 2)\left(\left(a_{1} / b_{1}\right) \tau_{1}^{2}-\left(2 / b_{1}\right) \tau_{1} \omega_{1}+\left(d_{1} / b_{1}\right) \omega_{1}^{2}-\pi / 2\right)} f\left(\mathbf{x}+\frac{\boldsymbol{\tau}}{2}\right)\right|^{p} d \boldsymbol{\tau}\right)^{1 / p} \\
& \quad \times\left(\int_{\mathbb{R}^{2}} \mid \bar{g}\left(\mathbf{x}-\frac{\boldsymbol{\tau}}{2}\right) \frac{1}{\sqrt{2 \pi b_{2}}}\right. \\
& \left.\left.\quad \cdot e^{(\mathbf{j} / 2)\left(\left(a_{2} / b_{2}\right) \tau_{2}^{2}-\left(2 / b_{2}\right) \tau_{2} \omega_{2}+\left(d_{2} / b_{2}\right) \omega_{2}^{2}-\pi / 2\right)}\right|^{q} d \boldsymbol{\tau}\right)^{1 / q} .
\end{aligned}
$$

Letting $\mathbf{x}+\boldsymbol{\tau} / 2=\mathbf{y}$ and $\mathbf{x}-\boldsymbol{\tau} / 2=\mathbf{z}$, we immediately obtain

$$
\begin{aligned}
& \left|\mathscr{W}_{f, g}^{A_{1}, A_{2}}(\mathbf{x}, \boldsymbol{\omega})\right| \\
& \quad=\frac{1}{2 \pi \sqrt{b_{1} b_{2}}}\left(\int_{\mathbb{R}^{2}}\left|f\left(\mathbf{x}+\frac{\boldsymbol{\tau}}{2}\right)\right|^{p} d \boldsymbol{\tau}\right)^{1 / p} \\
& \quad \cdot\left(\int_{\mathbb{R}^{2}}\left|\bar{g}\left(\mathbf{x}-\frac{\boldsymbol{\tau}}{2}\right)\right|^{q} d \boldsymbol{\tau}\right)^{1 / q} \\
& \quad=\frac{1}{2 \pi \sqrt{b_{1} b_{2}}}\left(\int_{\mathbb{R}^{2}}|f(\mathbf{y})|^{p} d \mathbf{y}\right)^{1 / p} \\
& \quad \cdot\left(\int_{\mathbb{R}^{2}}|\bar{g}(\mathbf{z})|^{q} d \mathbf{z}\right)^{1 / q} .
\end{aligned}
$$

Hence, the result follows.

Observe first that, for $p=q=2$, (34) will reduce to

$$
\left|\mathscr{W}_{f, g}^{A_{1}, A_{2}}(\mathbf{x}, \boldsymbol{\omega})\right| \leq \frac{1}{2 \pi \sqrt{b_{1} b_{2}}}\|f\|\|g\|,
$$

which shows that $\mathscr{W}_{f, g}^{A_{1}, A_{2}}(\mathbf{x}, \boldsymbol{\omega})$ is bounded on $L^{2}\left(\mathbb{R}^{2} ; \llbracket\right)$.

Lemma 11. The cross $Q W V D-L C T$ of a signal $f$ with matrix parameters $A_{1}=\left(a_{1}, b_{1}, c_{1}, d_{1}\right)$ and $A_{2}=\left(a_{2}, b_{2}, c_{2}, d_{2}\right)$ 
can be reduced as the QFT of the signal $f$ in the following form:

$$
\begin{aligned}
& \mathscr{W}_{f, g}^{A_{1}, A_{2}}(\mathbf{x}, \boldsymbol{\omega})=\frac{e^{-\mathbf{i}(\pi / 4)}}{\sqrt{2 \pi b_{1}}} \\
& \cdot e^{\left(\mathbf{i} d_{1} / 2 b_{1}\right) \omega_{1}^{2}} \mathscr{F}_{q}\left\{e^{\mathbf{i}\left(a_{1} / 2 b_{1}\right) \tau_{1}^{2}} f\left(\mathbf{x}+\frac{\boldsymbol{\tau}}{2}\right) \bar{g}\left(\mathbf{x}-\frac{\boldsymbol{\tau}}{2}\right)\right. \\
&\left.\cdot e^{\mathbf{j}\left(a_{2} / 2 b_{2}\right) \tau_{2}^{2}}\right\}\left(\frac{\omega_{1}}{b_{1}}, \frac{\omega_{2}}{b_{2}}\right) \frac{e^{-\mathbf{j}(\pi / 4)}}{\sqrt{2 \pi b_{2}}} e^{\left(\mathbf{j} d_{2} / 2 b_{2}\right) \omega_{2}^{2}} .
\end{aligned}
$$

Proof. By calculation directly, we get

$$
\begin{aligned}
& \mathscr{W}_{f, g}^{A_{1}, A_{2}}(\mathbf{x}, \boldsymbol{\omega})=\frac{1}{\sqrt{2 \pi b_{1}}} \\
& \cdot \int_{\mathbb{R}^{2}} e^{\mathbf{i}(1 / 2)\left(\left(a_{1} / b_{1}\right) \tau_{1}^{2}-\left(2 / b_{1}\right) \tau_{1} \omega_{1}+\left(d_{1} / b_{1}\right) \omega_{1}^{2}-\pi / 2\right)} f(\mathbf{x} \\
& \left.+\frac{\tau}{2}\right) \bar{g}\left(\mathbf{x}-\frac{\tau}{2}\right) \\
& \cdot \frac{1}{\sqrt{2 \pi b_{2}}} e^{\mathbf{j}(1 / 2)\left(\left(a_{2} / b_{2}\right) \tau_{2}^{2}-\left(2 / b_{2}\right) \tau_{2} \omega_{2}+\left(d_{2} / b_{2}\right) \omega_{2}^{2}-\pi / 2\right)} d \boldsymbol{\tau} \\
& =\frac{e^{-\mathbf{i}(\pi / 4)}}{\sqrt{2 \pi b_{1}}} \\
& \cdot e^{\mathbf{i}\left(d_{1} / 2 b_{1}\right) \omega_{1}^{2}} \int_{\mathbb{R}^{2}} e^{-\mathbf{i} \tau_{1}\left(\omega_{1} / b_{1}\right)}\left(e^{\mathbf{i}\left(a_{1} / 2 b_{1}\right) \tau_{1}^{2}} f\left(\mathbf{x}+\frac{\boldsymbol{\tau}}{2}\right)\right. \\
& \left.\cdot \bar{g}\left(\mathbf{x}-\frac{\boldsymbol{\tau}}{2}\right) e^{\mathbf{j}\left(a_{2} / 2 b_{2}\right) \tau_{2}^{2}}\right) e^{-\mathbf{j} \tau_{2}\left(\omega_{2} / b_{2}\right)} d \boldsymbol{\tau} \\
& \cdot \frac{e^{-\mathbf{j}(\pi / 4)}}{\sqrt{2 \pi b_{2}}} e^{\mathbf{j}\left(d_{2} / 2 b_{2}\right) \omega_{2}^{2}}=\frac{e^{-\mathbf{i}(\pi / 4)}}{\sqrt{2 \pi b_{1}}} \\
& \cdot e^{\left(\mathbf{i} d_{1} / 2 b_{1}\right) \omega_{1}^{2}} \mathscr{F}_{q}\left\{e^{\mathbf{i}\left(a_{1} / 2 b_{1}\right) \tau_{1}^{2}} f\left(\mathbf{x}+\frac{\boldsymbol{\tau}}{2}\right) \bar{g}\left(\mathbf{x}-\frac{\boldsymbol{\tau}}{2}\right)\right. \\
& \left.\cdot e^{\mathbf{j}\left(a_{2} / 2 b_{2}\right) \tau_{2}^{2}}\right\}\left(\frac{\omega_{1}}{b_{1}}, \frac{\omega_{2}}{b_{2}}\right) \frac{e^{-\mathbf{j}(\pi / 4)}}{\sqrt{2 \pi b_{2}}} e^{\left(\mathbf{j} d_{2} / 2 b_{2}\right) \omega_{2}^{2}},
\end{aligned}
$$

where the last line follows directly from (28).

For abbreviation, we use the notation

$$
\begin{aligned}
\widetilde{h_{f, g}}(\mathbf{x}, \boldsymbol{\tau})= & \frac{e^{-\mathbf{i}(\pi / 4)}}{\sqrt{2 \pi b_{1}}} e^{\mathbf{i}\left(a_{1} / 2 b_{1}\right) \tau_{1}^{2}} f\left(\mathbf{x}+\frac{\boldsymbol{\tau}}{2}\right) \bar{g}\left(\mathbf{x}-\frac{\boldsymbol{\tau}}{2}\right) \\
& \cdot e^{\mathbf{j}\left(a_{2} / 2 b_{2}\right) \tau_{2}^{2}} \frac{e^{-\mathbf{j}(\pi / 4)}}{\sqrt{2 \pi b_{2}}} .
\end{aligned}
$$

Therefore, we can write (38) in the form

$$
\begin{aligned}
\mathscr{F}_{q} & \left\{\widetilde{h_{f, g}}(\mathbf{x}, \boldsymbol{\tau})\right\}\left(\frac{\omega_{1}}{b_{1}}, \frac{\omega_{2}}{b_{2}}\right) \\
= & e^{-\left(\mathbf{i} d_{1} / 2 b_{1}\right) \omega_{1}^{2}} \mathscr{W}_{f, g}^{A_{1}, A_{2}}(\mathbf{x}, \boldsymbol{\omega}) e^{-\left(\mathbf{j} d_{2} / 2 b_{2}\right) \omega_{2}^{2}} .
\end{aligned}
$$

In the below theorem by combining the properties of the QFT and the fundamental relation between the QFT and the QWVD-LCT, we provide a new proof of reconstruction formula for the QWVD-LCT.

Theorem 12 (reconstruction formula for QWVD-LCT). The inverse transform of the cross the QWVD-LCT of the signal $f \in$ $L^{2}\left(\mathbb{R}^{2} ; \mathbb{H}\right)$ is given by

$$
\begin{aligned}
& f(x)=\frac{1}{\bar{g}(\mathbf{0})} \int_{\mathbb{R}^{2}} K_{A_{1}^{-1}}\left(x_{1}, \omega_{1}\right) \mathscr{W}_{f, g}^{A_{1}, A_{2}}\left(\frac{\mathbf{x}}{2}, \boldsymbol{\omega}\right) \\
& \cdot K_{A_{2}^{-1}}\left(x_{2}, \omega_{2}\right) d \boldsymbol{\omega}
\end{aligned}
$$

provided that $\bar{g}(\mathbf{0}) \neq \mathbf{0}$.

Proof. From the inverse transform of the QFT (17), it follows that

$$
\begin{gathered}
\widetilde{h_{f, g}}(\mathbf{x}, \boldsymbol{\tau})=\frac{1}{(2 \pi)^{2}} \int_{\mathbb{R}^{2}} e^{\mathbf{i}\left(\omega_{1} \tau_{1} / b_{1}\right)} \mathscr{F}_{q}\left\{\widetilde{h_{f, g}}(\mathbf{x}, \boldsymbol{\tau})\right\} \\
\cdot\left(\frac{\omega_{1}}{b_{1}}, \frac{\omega_{2}}{b_{2}}\right) e^{\mathbf{j}\left(\omega_{2} \tau_{2} / b_{2}\right)} d \frac{\boldsymbol{\omega}}{\mathbf{b}} .
\end{gathered}
$$

In view of (40) and (41), we easily get

$$
\begin{aligned}
& \frac{e^{-\mathbf{i}(\pi / 4)}}{\sqrt{2 \pi b_{1}}} e^{\mathbf{i}\left(a_{1} / 2 b_{1}\right) \tau_{1}^{2}} f\left(\mathbf{x}+\frac{\boldsymbol{\tau}}{2}\right) \bar{g}\left(\mathbf{x}-\frac{\boldsymbol{\tau}}{2}\right) e^{\mathbf{j}\left(a_{2} / 2 b_{2}\right) \tau_{2}^{2}} \\
& \cdot \frac{e^{-\mathbf{j}(\pi / 4)}}{\sqrt{2 \pi b_{2}}}=\frac{1}{(2 \pi)^{2}} \int_{\mathbb{R}^{2}} e^{\mathbf{i}\left(\omega_{1} \tau_{1} / b_{1}\right)} \mathscr{F}_{q}\left\{\widetilde{h_{f, g}}(\mathbf{x}, \boldsymbol{\tau})\right\} \\
& \cdot\left(\frac{\omega_{1}}{b_{1}}, \frac{\omega_{2}}{b_{2}}\right) e^{\mathbf{j}\left(\omega_{2} \tau_{2} / b_{2}\right)} d \frac{\boldsymbol{\omega}}{\mathbf{b}}=\frac{1}{(2 \pi)^{2}} \\
& \cdot \int_{\mathbb{R}^{2}} e^{\mathbf{i}\left(\omega_{1} \tau_{1} / b_{1}\right)} e^{-\left(\mathbf{i} d_{1} / 2 b_{1}\right) \omega_{1}^{2}} \mathscr{W}_{f, g}^{A_{1}, A_{2}}(\mathbf{x}, \boldsymbol{\omega}) \\
& \cdot e^{-\left(\mathbf{j} d_{2} / 2 b_{2}\right) \omega_{2}^{2}} e^{\mathbf{j}\left(\omega_{2} \tau_{2} / b_{2}\right)} d \frac{\boldsymbol{\omega}}{\mathbf{b}} .
\end{aligned}
$$

Subsequently,

$$
\begin{aligned}
& f\left(\mathbf{x}+\frac{\boldsymbol{\tau}}{2}\right) \bar{g}\left(\mathbf{x}-\frac{\boldsymbol{\tau}}{2}\right) \\
& =\int_{\mathbb{R}^{2}} \frac{e^{\mathbf{i}(\pi / 4)}}{\sqrt{2 \pi b_{1}}} e^{-\mathbf{i}\left(a_{1} / 2 b_{1}\right) \tau_{1}^{2}} e^{\mathbf{i}\left(\omega_{1} \tau_{1} / b_{1}\right)} e^{-\left(\mathbf{i} d_{1} / 2 b_{1}\right) \omega_{1}^{2}} \mathscr{W}_{f, \mathcal{g}}^{A_{1}, A_{2}}(\mathbf{x}, \boldsymbol{\omega}) \\
& \quad \cdot \frac{e^{\mathbf{j}(\pi / 4)}}{\sqrt{2 \pi b_{2}}} e^{-\left(\mathbf{j} d_{2} / 2 b_{2}\right) \omega_{2}^{2}} e^{\mathbf{j}\left(\omega_{2} \tau_{2} / b_{2}\right)} e^{-\mathbf{j}\left(a_{2} / 2 b_{2}\right) \tau_{2}^{2}} d \boldsymbol{\omega} \\
& =\int_{\mathbb{R}^{2}} \frac{1}{\sqrt{2 \pi b_{1}}} e^{-(\mathbf{i} / 2)\left(\left(a_{1} / b_{1}\right) \tau_{1}^{2}-\left(2 / b_{1}\right) \tau_{1} \omega_{1}+\left(d_{1} / b_{1}\right) \omega_{1}^{2}-\pi / 2\right)} \mathscr{W}_{f, \mathcal{g}}^{A_{1}, A_{2}}(\mathbf{x}, \\
& \quad \boldsymbol{\omega}) \frac{1}{\sqrt{2 \pi b_{2}}} e^{-(\mathbf{j} / 2)\left(\left(a_{2} / b_{2}\right) \tau_{2}^{2}-\left(2 / b_{2}\right) \tau_{2} \omega_{2}+\left(d_{2} / b_{2}\right) \omega_{2}^{2}-\pi / 2\right)} d \boldsymbol{\omega} \\
& =\int_{\mathbb{R}^{2}} K_{A_{1}^{-1}}\left(\tau_{1}, \omega_{1}\right) \mathscr{W}_{f, g}^{A_{1}, A_{2}}(\mathbf{x}, \boldsymbol{\omega}) K_{A_{2}^{-1}}\left(\tau_{2}, \omega_{2}\right) d \boldsymbol{\omega} .
\end{aligned}
$$


Letting $\boldsymbol{\tau} / 2=\mathbf{x}$, the above expression will lead to

$$
\begin{aligned}
& f(2 \mathbf{x}) \bar{g}(\mathbf{0})=\int_{\mathbb{R}^{2}} K_{A_{1}^{-1}}\left(2 x_{1}, \omega_{1}\right) \mathscr{W}_{f, g}^{A_{1}, A_{2}}(\mathbf{x}, \boldsymbol{\omega}) \\
& \cdot K_{A_{2}^{-1}}\left(2 x_{2}, \omega_{2}\right) d \boldsymbol{\omega}
\end{aligned}
$$

and the final result can be obtained by letting $2 \mathbf{x}=\mathbf{s}$; that is,

$$
\begin{aligned}
& f(\mathbf{s})=\frac{1}{\bar{g}(\mathbf{0})} \int_{\mathbb{R}^{2}} K_{A_{1}^{-1}}\left(s_{1}, \omega_{1}\right) \mathscr{W}_{f, g}^{A_{1}, A_{2}}\left(\frac{\mathbf{s}}{2}, \boldsymbol{\omega}\right) \\
& \cdot K_{A_{2}^{-1}}\left(s_{2}, \omega_{2}\right) d \boldsymbol{\omega},
\end{aligned}
$$

which completes the proof.

We now observe that from (45), we straightforwardly obtain the time marginal property of the QWVD-LCT; that is,

$$
\begin{aligned}
& f\left(\mathbf{x}+\frac{\boldsymbol{\tau}}{2}\right) \bar{g}\left(\mathbf{x}-\frac{\boldsymbol{\tau}}{2}\right) \\
& \quad=\int_{\mathbb{R}^{2}} \frac{1}{\sqrt{2 \pi b_{1}}} e^{(\mathbf{i} / 2)\left(\left(a_{1} / b_{1}\right) \tau_{1}^{2}-\left(2 / b_{1}\right) \tau_{1} \omega_{1}+\left(d_{1} / b_{1}\right) \omega_{1}^{2}-\pi / 2\right)} \mathscr{W}_{f, \mathcal{g}}^{A_{1}, A_{2}}(\mathbf{x}, \\
& \text { w) } \frac{1}{\sqrt{2 \pi b_{2}}} e^{(\mathbf{j} / 2)\left(\left(a_{2} / b_{2}\right) \tau_{2}^{2}-\left(2 / b_{2}\right) \tau_{2} \omega_{2}+\left(d_{2} / b_{2}\right) \omega_{2}^{2}-\pi / 2\right)} d \boldsymbol{\omega} .
\end{aligned}
$$

If we set $\boldsymbol{\tau}=0$, the above identity will reduce to

$$
\begin{gathered}
f(\mathbf{x}) \bar{g}(\mathbf{x})=\int_{\mathbb{R}^{2}} \frac{e^{\mathbf{i}(\pi / 4)}}{\sqrt{2 \pi b_{1}}} e^{-\mathbf{i}\left(d_{1} / 2 b_{1}\right) \omega_{1}^{2}} \mathscr{W}_{f, g}^{A_{1}, A_{2}}(\mathbf{x}, \boldsymbol{\omega}) \\
\cdot \frac{e^{\mathbf{j}(\pi / 4)}}{\sqrt{2 \pi b_{2}}} e^{-\mathbf{j}\left(d_{2} / 2 b_{2}\right) \omega_{2}^{2}} d \boldsymbol{\omega},
\end{gathered}
$$

and if we set $\tau=0$ and $f=g$, (48) becomes

$$
\begin{gathered}
|f(\mathbf{x})|^{2}=\int_{\mathbb{R}^{2}} \frac{e^{\mathbf{i}(\pi / 4)}}{\sqrt{2 \pi b_{1}}} e^{-\mathbf{i}\left(d_{1} / 2 \mathrm{~b}_{1}\right) \omega_{1}^{2}} \mathscr{W}_{f}^{A_{1}, A_{2}}(\mathbf{x}, \boldsymbol{\omega}) \\
\cdot \frac{e^{\mathbf{j}(\pi / 4)}}{\sqrt{2 \pi b_{2}}} e^{-\mathbf{j}\left(d_{2} / 2 b_{2}\right) \omega_{2}^{2}} d \boldsymbol{\omega} .
\end{gathered}
$$

Integrating (49) and (50) with respect to the $\mathbf{x}$-variable gives

$$
\begin{aligned}
\int_{\mathbb{R}^{2}} f(\mathbf{x}) \bar{g}(\mathbf{x}) d \mathbf{x} \\
=\int_{\mathbb{R}^{2}} \int_{\mathbb{R}^{2}} \frac{e^{\mathbf{i}(\pi / 4)}}{\sqrt{2 \pi b_{1}}} e^{-\mathbf{i}\left(d_{1} / 2 b_{1}\right) \omega_{1}^{2} \mathscr{W}_{f, g}^{A_{1}, A_{2}}(\mathbf{x}, \boldsymbol{\omega})} \\
\cdot \frac{e^{\mathbf{j}(\pi / 4)}}{\sqrt{2 \pi b_{2}}} e^{-\mathbf{j}\left(d_{2} / 2 b_{2}\right) \omega_{2}^{2}} d \boldsymbol{\omega} d \mathbf{x}
\end{aligned}
$$

$$
\begin{aligned}
\int_{\mathbb{R}^{2}}|f(\mathbf{x})|^{2} d \mathbf{x} \\
=\int_{\mathbb{R}^{2}} \int_{\mathbb{R}^{2}} \frac{e^{\mathbf{i}(\pi / 4)}}{\sqrt{2 \pi b_{1}}} e^{-\mathbf{i}\left(d_{1} / 2 b_{1}\right) \omega_{1}^{2}} \mathscr{W}_{f}^{A_{1}, A_{2}}(\mathbf{x}, \boldsymbol{\omega}) \\
\cdot \frac{e^{\mathbf{j}(\pi / 4)}}{\sqrt{2 \pi b_{2}}} e^{-\mathbf{j}\left(d_{2} / 2 b_{2}\right) \omega_{2}^{2}} d \boldsymbol{\omega} d \mathbf{x} .
\end{aligned}
$$

Applying Moyal's formula for the QFT and the relation between the QFT and the QWVD-LCT, we establish general Moyal's formula of the QWVD-LCT (compared to [9]).

Theorem 13 (Moyal's formula for the QWVD-LCT). Let $f_{1}, f_{2}, g_{1}, g_{2} \in L^{2}\left(\mathbb{R}^{2} ; \mathbb{H}\right)$ be quaternion-valued signals. Then the following equation holds:

$$
\begin{aligned}
\mathrm{Sc} & \int_{\mathbb{R}^{2}} \int_{\mathbb{R}^{2}} \mathscr{W}_{f_{1}, g_{1}}^{A_{1}, A_{2}}(\mathbf{x}, \boldsymbol{\omega}) \overline{\mathscr{W}_{f_{2}, g_{2}}^{A_{1}, A_{2}}(\mathbf{x}, \boldsymbol{\omega})} d \boldsymbol{\omega} d \boldsymbol{\tau} \\
& =\left\langle f_{1}\left(\overline{g_{1}}, \overline{g_{2}}\right), f_{2}\right\rangle .
\end{aligned}
$$

Proof. Applying Parseval's formula of the QFT (18) to $\boldsymbol{\omega}$ integral into the left-hand side of (52) yields

$$
\begin{aligned}
& \int_{\mathbb{R}^{2}}\left\langle\widetilde{h_{f_{1}, g_{1}}}, \widetilde{h_{f_{2}, g_{2}}}\right\rangle d \tau \\
& =\int_{\mathbb{R}^{2}}\left\langle\mathscr{F}_{q}\left\{\widetilde{h_{f_{1}, g_{1}}}\right\}, \mathscr{F}_{q}\left\{\widetilde{h_{f_{2}, g_{2}}}\right\}\right\rangle d \boldsymbol{\tau}=\frac{1}{\left|b_{1} b_{2}\right|} \\
& \cdot \int_{\mathbb{R}^{2}} \operatorname{Sc} \int_{\mathbb{R}^{2}} \mathscr{F}_{q}\left\{\widetilde{h_{f_{1}, g_{1}}}\right\}\left(\frac{\boldsymbol{\omega}}{\mathbf{b}}\right) \\
& \overline{\mathscr{F}_{q}\left\{\widetilde{\left.h_{f_{2}, g_{2}}\right\}}\left(\frac{\boldsymbol{\omega}}{\mathbf{b}}\right)\right.} d \boldsymbol{\omega} d \boldsymbol{\tau} .
\end{aligned}
$$

Using the cyclic multiplication symmetry (11) yields

$$
\begin{aligned}
& \int_{\mathbb{R}^{2}}\left\langle\widetilde{h_{f_{1}, g_{1}}}, \widetilde{h_{f_{2}, g_{2}}}\right\rangle d \tau=\frac{1}{\left|b_{1} b_{2}\right|} \\
& \cdot \operatorname{Sc} \int_{\mathbb{R}^{2}} \int_{\mathbb{R}^{2}} e^{-\mathbf{i}\left(d_{1} / 2 b_{1}\right) \omega_{1}^{2}} \mathscr{W}_{f_{1}, g_{1}}^{A_{1}, A_{2}}(\mathbf{x}, \boldsymbol{\omega}) \\
& \cdot e^{-\mathbf{j}\left(d_{2} / 2 b_{2}\right) \omega_{2}^{2}} e^{-\mathbf{i}\left(d_{1} / 2 b_{1}\right) \omega_{1}^{2} \mathscr{W}_{f_{2}, g_{2}}^{A_{1}, A_{2}}(\mathbf{x}, \boldsymbol{\omega}) e^{-\mathbf{j}\left(d_{2} / 2 b_{2}\right) \omega_{2}^{2}}} d \boldsymbol{\omega} d \boldsymbol{\tau} \\
& =\frac{1}{\left|b_{1} b_{2}\right|} \operatorname{Sc} \int_{\mathbb{R}^{2}} \int_{\mathbb{R}^{2}} e^{-\mathbf{i}\left(d_{1} / 2 b_{1}\right) \omega_{1}^{2}} \mathscr{W}_{f_{1}, g_{1}}^{A_{1}, A_{2}}(\mathbf{x}, \boldsymbol{\omega}) \\
& \cdot e^{-\mathbf{j}\left(d_{2} / 2 b_{2}\right) \omega_{2}^{2}} e^{\mathbf{j}\left(d_{2} / 2 b_{2}\right) \omega_{1}^{2}} \overline{\mathscr{W}_{f_{2}, g_{2}}^{A_{1}, A_{2}}(\mathbf{x}, \boldsymbol{\omega})} e^{\mathbf{i}\left(d_{1} / 2 b_{1}\right) \omega_{1}^{2}} d \boldsymbol{\omega} d \boldsymbol{\tau} \\
& =\frac{1}{\left|b_{1} b_{2}\right|} \operatorname{Sc} \int_{\mathbb{R}^{2}} \int_{\mathbb{R}^{2}} \mathscr{W}_{f_{1}, g_{1}}^{A_{1}, A_{2}}(\mathbf{x}, \boldsymbol{\omega}) \overline{\mathscr{W}_{f_{2}, g_{2}}^{A_{1}, A_{2}}(\mathbf{x}, \boldsymbol{\omega})} d \boldsymbol{\omega} d \boldsymbol{\tau} \text {. }
\end{aligned}
$$

The right-hand side of the above identity can be rewritten in the form 


$$
\begin{aligned}
& \int_{\mathbb{R}^{2}}\left\langle\widetilde{h_{f_{1}, g_{1}}}, \widetilde{h_{f_{2}, g_{2}}}\right\rangle d \boldsymbol{\tau}=\operatorname{Sc} \int_{\mathbb{R}^{2}} \int_{\mathbb{R}^{2}} \widetilde{h_{f_{1}, g_{1}}}(\mathbf{x}, \boldsymbol{\tau}) \overline{\overline{h_{f_{2}, g_{2}}}}(\mathbf{x}, \boldsymbol{\tau}) d \mathbf{x} d \boldsymbol{\tau} \\
& =\operatorname{Sc}\left(\int_{\mathbb{R}^{2}} \int_{\mathbb{R}^{2}} \frac{e^{-\mathbf{i}(\pi / 4)}}{\sqrt{2 \pi b_{1}}} e^{\mathbf{i}\left(a_{1} / 2 b_{1}\right) \tau_{1}^{2}} f_{1}\left(\mathbf{x}+\frac{\boldsymbol{\tau}}{2}\right) \overline{g_{1}}\left(\mathbf{x}-\frac{\boldsymbol{\tau}}{2}\right) e^{\mathbf{j}\left(a_{2} / 2 b_{2}\right) \tau_{2}^{2}} \frac{e^{-\mathbf{j}(\pi / 4)}}{\sqrt{2 \pi b_{2}}}\right. \\
& \left.\times \frac{\overline{e^{-\mathbf{i}(\pi / 4)}}}{\sqrt{2 \pi b_{1}}} e^{\mathrm{i}\left(a_{1} / 2 b_{1}\right) \tau_{1}^{2}} f_{2}\left(\mathbf{x}+\frac{\boldsymbol{\tau}}{2}\right) \overline{g_{2}}\left(\mathbf{x}-\frac{\boldsymbol{\tau}}{2}\right) \frac{e^{-\mathbf{j}(\pi / 4)}}{\sqrt{2 \pi b_{2}}} e^{\mathbf{j}\left(a_{2} / 2 b_{2}\right) \tau_{2}^{2}} d \mathbf{x} d \boldsymbol{\tau}\right) \\
& =\frac{1}{\left|b_{1} b_{2}\right|} \mathrm{Sc} \int_{\mathbb{R}^{2}} \int_{\mathbb{R}^{2}} f_{1}\left(\mathbf{x}+\frac{\boldsymbol{\tau}}{2}\right) \overline{g_{1}}\left(\mathbf{x}-\frac{\boldsymbol{\tau}}{2}\right) \overline{f_{2}\left(\mathbf{x}+\frac{\boldsymbol{\tau}}{2}\right) \overline{g_{2}}\left(\mathbf{x}-\frac{\boldsymbol{\tau}}{2}\right)} d \mathbf{x} d \boldsymbol{\tau} .
\end{aligned}
$$

Making the change of variables $\mathbf{y}=\mathbf{x}+\boldsymbol{\tau} / 2$ and $\mathbf{z}=\mathbf{x}-\boldsymbol{\tau} / 2$ and applying Fubini's theorem we obtain

$$
\begin{aligned}
\int_{\mathbb{R}^{2}} & \left\langle\widetilde{h_{f_{1}, g_{1}}} \widetilde{h_{f_{2}, g_{2}}}\right\rangle d \boldsymbol{\tau} \\
= & \frac{1}{\left|b_{1} b_{2}\right|} S c \int_{\mathbb{R}^{2}} \int_{\mathbb{R}^{2}} f_{1}(\mathbf{y}) \overline{g_{1}}(\mathbf{z}) g_{2}(\mathbf{z}) \overline{f_{2}}(\mathbf{y}) d \mathbf{y} d \mathbf{z} \\
= & \frac{1}{\left|b_{1} b_{2}\right|} S c \int_{\mathbb{R}^{2}} f_{1}(\mathbf{y}) \int_{\mathbb{R}^{2}} \overline{g_{1}}(\mathbf{z}) g_{2}(\mathbf{z}) d \mathbf{z} \overline{f_{2}}(\mathbf{y}) d \mathbf{y} \\
= & \frac{1}{\left|b_{1} b_{2}\right|}\left\langle f_{1}\left(\overline{g_{1}}, \overline{g_{2}}\right), f_{2}\right\rangle .
\end{aligned}
$$

By comparing the last line of (54) with the last line of (56) finishes the proof of the theorem.

Based on the above theorem, we obtain the following consequences:

(i) If $g_{1}=g_{2}$, then

$$
\begin{aligned}
& \operatorname{Sc} \int_{\mathbb{R}^{2}} \int_{\mathbb{R}^{2}} \mathscr{W}_{f_{1}, g_{1}}^{A_{1}, A_{2}}(\mathbf{x}, \boldsymbol{\omega}) \overline{\mathscr{W}_{f_{2}, g_{1}}^{A_{1}, A_{2}}(\mathbf{x}, \boldsymbol{\omega})} d \boldsymbol{\omega} d \boldsymbol{\tau} \\
& \quad=\left\|g_{1}\right\|^{2}\left\langle f_{1}, f_{2}\right\rangle .
\end{aligned}
$$

(ii) If $f_{1}=f_{2}$, then

$$
\begin{aligned}
\mathrm{Sc} & \int_{\mathbb{R}^{2}} \int_{\mathbb{R}^{2}} \mathscr{W}_{f_{1}, g_{1}}^{A_{1}, A_{2}}(\mathbf{x}, \boldsymbol{\omega}) \overline{\mathscr{W}_{f_{1}, g_{2}}^{A_{1}, A_{2}}(\mathbf{x}, \boldsymbol{\omega})} d \boldsymbol{\omega} d \boldsymbol{\tau} \\
\quad & \left\langle f_{1}\left(\overline{g_{1}}, \overline{g_{2}}\right), f_{1}\right\rangle .
\end{aligned}
$$

(iii) If $f_{1}=f_{2}$ and $g_{1}=g_{2}$, then

$$
\int_{\mathbb{R}^{2}} \int_{\mathbb{R}^{2}}\left|\mathscr{W}_{f_{1}, g_{1}}^{A_{1}, A_{2}}(\mathbf{x}, \boldsymbol{\omega})\right|^{2} d \boldsymbol{\omega} d \boldsymbol{\tau}=\left\|f_{1}\right\|^{2}\left\|g_{1}\right\|^{2}
$$

3.2. Relationship between QAF-LCT and QWFT. In this subsection, we introduce the quaternionic windowed Fourier transform (QWFT) and obtain the relationship between the QAF-LCT and QWFT. Following [11, 12], we define the QWFT as follows.
Definition 14 (QWFT). Let $\phi \in L^{2}\left(\mathbb{R}^{2} ; \mathbb{-}\right)$ be a fixed nonzero quaternion window function. The QWFT of $f \in L^{2}\left(\mathbb{R}^{2} ; \mathbb{R}\right)$ with respect to $\phi$ is defined to be the quaternion function on phase space $\mathbb{R}^{2} \times \mathbb{R}^{2}$ given by

$$
\mathcal{S}_{f}(\mathbf{x}, \boldsymbol{\omega})=\int_{\mathbb{R}^{2}} e^{-\mathbf{i} \omega_{1} \tau_{1}} f(\mathbf{x}) \bar{g}(\mathbf{x}-\boldsymbol{\tau}) e^{-\mathbf{j} \omega_{2} \tau_{2}} d \boldsymbol{\tau} .
$$

Before presenting the main result of this subsection, let us introduce the following carrier definition and its properties (see $[13,14])$.

Definition 15 (carrier). Given two quaternions $p$ and $q$, we define the right $C_{r}$ and left $C_{l}$ carrier operators as

$$
\begin{aligned}
C_{r}(p) q & =q p, \\
q C_{l}(p) & =p q .
\end{aligned}
$$

Lemma 16. Carriers (61) above satisfy the following properties with $p \in \mathbb{U}$ :

$$
\begin{aligned}
& \overline{C_{r}(p)}=C_{l}(\bar{p}), \\
& \overline{C_{l}(p)}=C_{r}(\bar{p}) .
\end{aligned}
$$

We now describe the relationship between the QAF-LCT and the QWFT in the following theorem.

Theorem 17. The QAF-LCT of a quaternion signal $f \in$ $L^{2}\left(\mathbb{R}^{2} ; \mathbb{W}\right)$ can be expressed by the QWFT in the form

$$
\begin{aligned}
& \mathscr{A}_{f, g}^{A_{1}, A_{2}}\left(\frac{\mathbf{x}}{2}, \frac{\boldsymbol{\omega}}{2}\right)=\frac{4 e^{-\mathbf{i}(\pi / 4)}}{\sqrt{2 \pi b_{1}}} \\
& \cdot e^{\mathbf{i}\left(\left(d_{1} / 8 b_{1}\right) \omega_{1}^{2}+\left(1 / 2 b_{1}\right) \omega_{1} x_{1}-\left(3 a_{1} / 2 b_{1}\right) x_{1}^{2}\right)} \mathcal{S}_{f}\left(\mathbf{x}, \frac{\omega_{1}+2 a_{1} x_{1}}{b_{1}},\right. \\
& \left.\frac{\omega_{2}+2 a_{2} x_{2}}{b_{2}}\right) \times \frac{4 e^{-\mathbf{j}(\pi / 4)}}{\sqrt{2 \pi b_{2}}} \\
& \cdot e^{\mathbf{j}\left(\left(d_{2} / 8 b_{2}\right) \omega_{2}^{2}+\left(1 / 2 b_{2}\right) \omega_{2} x_{2}-\left(3 a_{2} / 2 b_{2}\right) x_{2}^{2}\right)} .
\end{aligned}
$$

Proof. According to the definition of QWVD-LCT (28), we obtain the following by making the change of variable: 


$$
\begin{aligned}
& \mathscr{A}_{f, \boldsymbol{g}}^{A_{1}, A_{2}}\left(\frac{\mathbf{x}}{2}, \frac{\boldsymbol{\omega}}{2}\right)=\frac{4}{\sqrt{2 \pi b_{1}}} \\
& \cdot \int_{\mathbb{R}^{2}} e^{\mathbf{i}\left(\left(a_{1} / 2 b_{1}\right) \tau_{1}^{2}-\left(1 / 2 b_{1}\right) \tau_{1} \omega_{1}+\left(d_{1} / 8 b_{1}\right) \omega_{1}^{2}-\pi / 2\right)} f\left(\frac{\boldsymbol{\tau}}{2}+\frac{\mathbf{x}}{2}\right) \bar{g}\left(\frac{\boldsymbol{\tau}}{2}-\frac{\mathbf{x}}{2}\right) \frac{1}{\sqrt{2 \pi b_{2}}} e^{\mathbf{j}\left(\left(a_{2} / 2 b_{2}\right) \tau_{2}^{2}-\left(1 / 2 b_{2}\right) \tau_{2} \omega_{2}+\left(d_{2} / 8 b_{2}\right) \omega_{2}^{2}-\pi / 2\right)} d \boldsymbol{\tau} \\
& =\frac{4 e^{-\mathbf{i}(\pi / 4)}}{\sqrt{2 \pi b_{1}}} e^{\left(\mathbf{i} d_{1} / 8 b_{1}\right) \omega_{1}^{2}} \int_{\mathbb{R}^{2}} e^{-\mathbf{i}\left(\tau_{1}-x_{1} / 2\right)\left(\omega_{1} / b_{1}\right)} e^{\mathbf{i}\left(2 a_{1} / b_{1}\right)\left(\tau_{1}-x_{1} / 2\right)^{2}} f(\boldsymbol{\tau}) \bar{g}(\boldsymbol{\tau}-\mathbf{x}) e^{\mathbf{j}\left(2 a_{2} / b_{2}\right)\left(\tau_{2}-x_{2} / 2\right)^{2}} e^{-\mathbf{j}\left(\tau_{2}-x_{2} / 2\right)\left(\omega_{2} / b_{2}\right)} d \boldsymbol{\tau} \\
& \quad \times \frac{4 e^{-\mathbf{j}(\pi / 4)}}{\sqrt{2 \pi b_{2}}} e^{\left(\mathbf{j} d_{2} / 8 b_{2}\right) \omega_{2}^{2}}=\frac{4 e^{-\mathbf{i}(\pi / 4)}}{\sqrt{2 \pi b_{1}}} e^{\mathbf{i}\left(\left(d_{1} / 8 b_{1}\right) \omega_{1}^{2}+\left(1 / 2 b_{1}\right) \omega_{1} x_{1}-\left(3 a_{1} / 2 b_{1}\right) x_{1}^{2}\right)} \int_{\mathbb{R}^{2}} e^{-\mathbf{i}\left(\left(\omega_{1}+2 a_{1} x_{1}\right) / b_{1}\right) \tau_{1}} e^{\mathbf{i}\left(2 a_{1} / b_{1}\right)\left(x_{1}-\tau_{1}\right)^{2}} f(\mathbf{x}) \bar{g}(\mathbf{x}-\boldsymbol{\tau}) \\
& \quad \times e^{\mathbf{j}\left(2 a_{2} / b_{2}\right)\left(x_{2}-\tau_{2}\right)^{2}} e^{-\mathbf{j}\left(\left(\omega_{2}+2 a_{2} x_{2}\right) / b_{2}\right) \tau_{2}} d \boldsymbol{\tau} \frac{4 e^{-\mathbf{j}(\pi / 4)}}{\sqrt{2 \pi b_{2}}} e^{\mathbf{j}\left(\left(d_{2} / 8 b_{2}\right) \omega_{2}^{2}+\left(1 / 2 b_{2}\right) \omega_{2} x_{2}-\left(3 a_{2} / 2 b_{2}\right) x_{2}^{2}\right)} .
\end{aligned}
$$

With the help of (61) and (62), we rewrite the above identity as

$$
\begin{aligned}
& \mathscr{A}_{f, \mathcal{G}}^{A_{1}, A_{2}}\left(\frac{\mathbf{x}}{2}, \frac{\boldsymbol{\omega}}{2}\right)=\frac{4 e^{-\mathbf{i}(\pi / 4)}}{\sqrt{2 \pi b_{1}}} \\
& \cdot e^{\mathbf{i}\left(\left(d_{1} / 8 b_{1}\right) \omega_{1}^{2}+\left(1 / 2 b_{1}\right) \omega_{1} x_{1}-\left(3 a_{1} / 2 b_{1}\right) x_{1}^{2}\right)} \int_{\mathbb{R}^{2}} e^{-\mathbf{i}\left(\left(\omega_{1}+2 a_{1} x_{1}\right) / b_{1}\right) \tau_{1}} f(\mathbf{x}) \\
& \times \overline{e^{-\mathbf{j}\left(2 a_{2} / b_{2}\right)\left(x_{2}-\tau_{2}\right)^{2}} g(\mathbf{x}-\boldsymbol{\tau}) \operatorname{Cr}\left(e^{-\mathbf{i}\left(2 a_{1} / b_{1}\right)\left(x_{1}-\tau_{1}\right)^{2}}\right)} \\
& \times e^{-\mathbf{j}\left(\left(\omega_{2}+2 a_{2} x_{2}\right) / b_{2}\right) \tau_{2}} d \boldsymbol{\tau} \frac{4 e^{-\mathbf{j}(\pi / 4)}}{\sqrt{2 \pi b_{2}}} \\
& \cdot e^{\mathbf{j}\left(\left(d_{2} / 8 b_{2}\right) \omega_{2}^{2}+\left(1 / 2 b_{2}\right) \omega_{2} x_{2}-\left(3 a_{2} / 2 b_{2}\right) x_{2}^{2}\right)} \text {. }
\end{aligned}
$$

According to the definition of QWFT (60), we choose the quaternion window function

$$
g(\mathbf{x})=e^{-\mathbf{j}\left(2 a_{2} / b_{2}\right) x_{2}^{2}} g(\mathbf{x}) \operatorname{Cr}\left(e^{-\mathbf{i}\left(2 a_{1} / b_{1}\right) x_{1}^{2}}\right)
$$

and obtain

$$
\begin{aligned}
& \mathscr{A}_{f, g}^{A_{1}, A_{2}}\left(\frac{\mathbf{x}}{2}, \frac{\boldsymbol{\omega}}{2}\right)=\frac{4 e^{-\mathbf{i}(\pi / 4)}}{\sqrt{2 \pi b_{1}}} \\
& \cdot e^{\mathbf{i}\left(\left(d_{1} / 8 b_{1}\right) \omega_{1}^{2}+\left(1 / 2 b_{1}\right) \omega_{1} x_{1}-\left(3 a_{1} / 2 b_{1}\right) x_{1}^{2}\right)} \mathcal{S}_{f}\left(\mathbf{x}, \frac{\omega_{1}+2 a_{1} x_{1}}{b_{1}},\right. \\
& \left.\frac{\omega_{2}+2 a_{2} x_{2}}{b_{2}}\right) \times \frac{4 e^{-\mathbf{j}(\pi / 4)}}{\sqrt{2 \pi b_{2}}} \\
& \cdot e^{\mathbf{j}\left(\left(d_{2} / 8 b_{2}\right) \omega_{2}^{2}+\left(1 / 2 b_{2}\right) \omega_{2} x_{2}-\left(3 a_{2} / 2 b_{2}\right) x_{2}^{2}\right)} .
\end{aligned}
$$

The proof is complete.
3.3. Relationship between QAF-LCT and CQWT. Before proving the relationship between the QAF-LCT and the continuous quaternion wavelet transform (CQWT), we first introduce the definition of the CQWT (see [15-17]).

Definition 18 (CQWT). The CQWT of a quaternion function $f \in L^{2}\left(\mathbb{R}^{2} ; \mathbb{H}\right)$ with respect to the quaternion mother wavelet $\psi$ is defined by

$$
T_{\psi} f(a, \mathbf{b})=\int_{\mathbb{R}^{2}} f(\mathbf{x}) \frac{1}{a} \overline{\psi\left(\frac{\mathbf{x}-\mathbf{b}}{a}\right)} d \mathbf{x} .
$$

Here the family of the quaternion wavelets $\psi_{a, \mathbf{b}}$ is defined by

$$
\psi_{a, \mathbf{b}}(\mathbf{x})=\frac{1}{a} \psi\left(\frac{\mathbf{x}-\mathbf{b}}{a}\right), \quad a \in \mathbb{R}^{+} .
$$

This definition will lead to the following result.

Theorem 19. The QAF-LCT of $f \in L^{2}\left(\mathbb{R}^{2} ; \mathbb{H}\right)$ can be reduced to the CQWT

$$
\begin{aligned}
& \mathscr{A}_{f, \mathcal{g}}^{A_{1}, A_{2}}(\mathbf{x}, \boldsymbol{\omega})=\frac{e^{-\mathbf{i}(\pi / 4)}}{\sqrt{2 \pi b_{1}}} \\
& \cdot e^{\mathbf{i}\left(d_{1} / 2 b_{1}\right) \omega_{1}^{2}-\omega_{1} x_{1} / 2 b_{1}+a_{1} x_{1}^{2} / 8 b_{1}} T_{\psi} f(1, \mathbf{x}) \frac{e^{-\mathbf{j}(\pi / 4)}}{\sqrt{2 \pi b_{2}}} \\
& \cdot e^{\mathbf{j}\left(d_{2} / 2 b_{2}\right) \omega_{2}^{2}-\omega_{2} x_{2} / 2 b_{2}+a_{2} x_{2}^{2} / 8 b_{2}} .
\end{aligned}
$$

Proof. Applying the definition of the QAF-LCT (29) and following [18], we easily get

$$
\begin{aligned}
& \mathscr{A}_{f, \boldsymbol{g}}^{A_{1}, A_{2}}(\mathbf{x}, \boldsymbol{\omega}) \\
& \quad=\frac{e^{-\mathbf{i}(\pi / 4)}}{\sqrt{2 \pi b_{1}}} \int_{\mathbb{R}^{2}} e^{\mathbf{i}\left(\left(a_{1} / 2 b_{1}\right) \tau_{1}^{2}-\left(1 / b_{1}\right) \tau_{1} \omega_{1}+\left(d_{1} / 2 b_{1}\right) \omega_{1}^{2}-\pi / 2\right)} f\left(\boldsymbol{\tau}+\frac{\mathbf{x}}{2}\right) \bar{g}\left(\boldsymbol{\tau}-\frac{\mathbf{x}}{2}\right) \frac{e^{-\mathbf{j}(\pi / 4)}}{\sqrt{2 \pi b_{2}}} e^{\mathbf{j}\left(\left(a_{2} / 2 b_{2}\right) \tau_{2}^{2}-\left(1 / b_{2}\right) \tau_{2} \omega_{2}+\left(d_{2} / 2 b_{2}\right) \omega_{2}^{2}-\pi / 2\right)} d \boldsymbol{\tau}
\end{aligned}
$$




$$
\begin{aligned}
& =\frac{e^{-\mathbf{i}(\pi / 4)}}{\sqrt{2 \pi b_{1}}} \int_{\mathbb{R}^{2}} e^{\mathbf{i}\left(\left(a_{1} / 2 b_{1}\right) \tau_{1}^{2}-\left(1 / b_{1}\right) \tau_{1} \omega_{1}+\left(d_{1} / 2 b_{1}\right) \omega_{1}^{2}-\pi / 2\right)} f\left(\boldsymbol{\tau}+\frac{\mathbf{x}}{2}\right) \bar{g}\left(\boldsymbol{\tau}+\frac{\mathbf{x}}{2}-\mathbf{x}\right) \frac{e^{-\mathbf{j}(\pi / 4)}}{\sqrt{2 \pi b_{2}}} e^{\mathbf{j}\left(\left(a_{2} / 2 b_{2}\right) \tau_{2}^{2}-\left(1 / b_{2}\right) \tau_{2} \omega_{2}+\left(d_{2} / 2 b_{2}\right) \omega_{2}^{2}-\pi / 2\right)} d \boldsymbol{\tau} \\
& =\frac{e^{-\mathbf{i}(\pi / 4)}}{\sqrt{2 \pi b_{1}}} \int_{\mathbb{R}^{2}} e^{\mathbf{i}\left(d_{1} / 2 b_{1}\right) \omega_{1}^{2}} e^{\mathbf{i}\left(1 / b_{1}\right)\left(\tau_{1}^{\prime}-x_{1} / 2\right) \omega_{1}} e^{\mathbf{i}\left(a_{1} / 2 b_{1}\right)\left(\tau_{1}^{\prime}-x_{1} / 2\right)^{2} \omega_{1}} f\left(\boldsymbol{\tau}^{\prime}\right) \bar{g}\left(\boldsymbol{\tau}^{\prime}-\mathbf{x}\right) \\
& \times \frac{e^{-\mathbf{j}(\pi / 4)}}{\sqrt{2 \pi b_{2}}} e^{\mathbf{j}\left(d_{2} / 2 b_{2}\right) \omega_{2}^{2}} e^{\mathbf{j}\left(1 / b_{2}\right)\left(\tau_{2}^{\prime}-x_{2} / 2\right) \omega_{2}} e^{\mathbf{j}\left(a_{2} / 2 b_{2}\right)\left(\tau_{2}^{\prime}-x_{2} / 2\right)^{2} \omega_{1}} d \boldsymbol{\tau}^{\prime} \\
& =\frac{e^{-\mathbf{i}(\pi / 4)}}{\sqrt{2 \pi b_{1}}} e^{\mathbf{i}\left(\left(d_{1} / 2 b_{1}\right) \omega_{1}^{2}-\omega_{1} x_{1} / 2 b_{1}+a_{1} x_{1}^{2} / 8 b_{1}\right)} \int_{\mathbb{R}^{2}} e^{\mathbf{i}\left(a_{1} / 2 b_{1}\right)\left(\tau_{1}^{\prime}-x_{1}\right)^{2}-\mathbf{i}\left(\tau_{1}^{\prime}-x_{1}\right)\left(\left(2 \omega_{1}-a_{1} x_{1}\right) / 2 b_{1}\right)} f\left(\boldsymbol{\tau}^{\prime}\right) \bar{g}\left(\boldsymbol{\tau}^{\prime}-\mathbf{x}\right) \\
& \times e^{\mathbf{j}\left(a_{2} / 2 b_{2}\right)\left(\tau_{2}^{\prime}-x_{2}\right)^{2}-\mathbf{j}\left(\tau_{2}^{\prime}-x_{2}\right)\left(\left(2 \omega_{2}-a_{2} x_{2}\right) / 2 b_{2}\right)} d \boldsymbol{\tau}^{\prime} \frac{e^{-\mathbf{j}(\pi / 4)}}{\sqrt{2 \pi b_{2}}} e^{\mathbf{j}\left(\left(d_{2} / 2 b_{2}\right) \omega_{2}^{2}-\omega_{2} x_{2} / 2 b_{2}+a_{2} x_{2}^{2} / 8 b_{2}\right)} .
\end{aligned}
$$

Again applying (61) and (62), we obtain

$$
\begin{aligned}
\mathscr{A}_{f, g}^{A_{1}, A_{2}}(\mathbf{x}, \boldsymbol{\omega})= & \frac{e^{-\mathbf{i}(\pi / 4)}}{\sqrt{2 \pi b_{1}}} e^{\mathbf{i}\left(\left(d_{1} / 2 b_{1}\right) \omega_{1}^{2}-\omega_{1} x_{1} / 2 b_{1}+a_{1} x_{1}^{2} / 8 b_{1}\right)} \int_{\mathbb{R}^{2}} f\left(\boldsymbol{\tau}^{\prime}\right) \\
& \times \overline{e^{-\mathbf{j}\left(a_{2} / 2 b_{2}\right)\left(\tau_{2}^{\prime}-x_{2}\right)^{2}+\mathbf{j}\left(\tau_{2}^{\prime}-x_{2}\right)\left(\left(2 \omega_{2}-a_{2} x_{2}\right) / 2 b_{2}\right)} g\left(\boldsymbol{\tau}^{\prime}-\mathbf{x}\right) \operatorname{Cr}\left(e^{\left.-\mathbf{i}\left(a_{1} / 2 b_{1}\right)\left(\tau_{1}^{\prime}-x_{1}\right)^{2}+\mathbf{i}\left(\tau_{1}^{\prime}-x_{1}\right)\left(\left(2 \omega_{1}-a_{1} x_{1}\right) / 2 b_{1}\right)\right)}\right.} \\
& \times d \boldsymbol{\tau}^{\prime} \frac{e^{-\mathbf{j}(\pi / 4)}}{\sqrt{2 \pi b_{2}}} e^{\mathbf{j}\left(\left(d_{2} / 2 b_{2}\right) \omega_{2}^{2}-\omega_{2} x_{2} / 2 b_{2}+a_{2} x_{2}^{2} / 8 b_{2}\right)} \\
= & \frac{e^{-\mathbf{i}(\pi / 4)}}{\sqrt{2 \pi b_{1}}} e^{\mathbf{i}\left(\left(d_{1} / 2 b_{1}\right) \omega_{1}^{2}-\omega_{1} x_{1} / 2 b_{1}+a_{1} x_{1}^{2} / 8 b_{1}\right)} \int_{\mathbb{R}^{2}} f\left(\boldsymbol{\tau}^{\prime}\right) \bar{\psi}\left(\boldsymbol{\tau}^{\prime}-\mathbf{x}\right) d \boldsymbol{\tau}^{\prime} \frac{e^{-\mathbf{j}(\pi / 4)}}{\sqrt{2 \pi b_{2}}} e^{\mathbf{j}\left(\left(d_{2} / 2 b_{2}\right) \omega_{2}^{2}-\omega_{2} x_{2} / 2 b_{2}+a_{2} x_{2}^{2} / 8 b_{2}\right)} \\
= & \frac{e^{-\mathbf{i}(\pi / 4)}}{\sqrt{2 \pi b_{1}}} e^{\mathbf{i}\left(\left(d_{1} / 2 b_{1}\right) \omega_{1}^{2}-\omega_{1} x_{1} / 2 b_{1}+a_{1} x_{1}^{2} / 8 b_{1}\right)} T_{\psi} f(1, \mathbf{x}) \frac{e^{-\mathbf{j}(\pi / 4)}}{\sqrt{2 \pi b_{2}}} e^{\mathbf{j}\left(\left(d_{2} / 2 b_{2}\right) \omega_{2}^{2}-\omega_{2} x_{2} / 2 b_{2}+a_{2} x_{2}^{2} / 8 b_{2}\right)},
\end{aligned}
$$

where the family of the quaternion wavelets $\psi_{1, \mathbf{b}}$ is given by

$$
\psi_{1, \mathbf{b}}(\mathbf{x})=\overline{e^{-\mathbf{j}\left(a_{2} / 2 b_{2}\right)\left(\tau_{2}-x_{2}\right)^{2}+\mathbf{j}\left(\tau_{2}-x_{2}\right)\left(\left(2 \omega_{2}-a_{2} x_{2}\right) / 2 b_{2}\right)} g(\boldsymbol{\tau}-\mathbf{x}) \operatorname{Cr}\left(e^{-\mathbf{i}\left(a_{1} / 2 b_{1}\right)\left(\tau_{1}-x_{1}\right)^{2}+\mathbf{i}\left(\tau_{1}-x_{1}\right)\left(\left(2 \omega_{1}-a_{1} x_{1}\right) / 2 b_{1}\right)}\right)} .
$$

This is the desired result.

\section{Relationship between QAF-LCT and QFT}

By a similar argument as in the proof of the Lemma 11, one may establish the following lemma, which describes the basic relationship between the QAF-LCT and the QFT.

Lemma 20. The cross QAF-LCT of a signal $f$ with matrix parameters $A_{1}=\left(a_{1}, b_{1}, c_{1}, d_{1}\right)$ and $A_{2}=\left(a_{2}, b_{2}, c_{2}, d_{2}\right)$ can be reduced as the QFT of the signal $f$ in the following form:

$$
\begin{aligned}
& \mathscr{A}_{f, \mathcal{G}}^{A_{1}, A_{2}}(\mathbf{x}, \boldsymbol{\omega})=\frac{e^{-\mathbf{i}(\pi / 4)}}{\sqrt{2 \pi b_{1}}} \\
& \cdot e^{\left(\mathbf{i} d_{1} / 2 b_{1}\right) \omega_{1}^{2}} \mathscr{F}_{q}\left\{e^{\mathbf{i}\left(a_{1} / 2 b_{1}\right) \tau_{1}^{2}} f\left(\boldsymbol{\tau}+\frac{\mathbf{x}}{2}\right) \bar{g}\left(\boldsymbol{\tau}-\frac{\mathbf{x}}{2}\right)\right. \\
& \left.\cdot e^{\mathbf{j}\left(a_{2} / 2 b_{2}\right) \tau_{2}^{2}}\right\}\left(\frac{\omega_{1}}{b_{1}}, \frac{\omega_{2}}{b_{2}}\right) \frac{e^{-\mathbf{j}(\pi / 4)}}{\sqrt{2 \pi b_{2}}} e^{\left(\mathbf{j} d_{2} / 2 b_{2}\right) \omega_{2}^{2}} .
\end{aligned}
$$


Applying (74) and inversion formula for the QFT (17), we easily obtain the following theorem.

Theorem 21 (reconstruction formula for QAF-LCT). The inverse transform of the cross the QAF-LCT of the signal $f \in$ $L^{2}\left(\mathbb{R}^{2} ; \mathbb{H}\right)$ is given by

$$
\begin{aligned}
& f(x)=\frac{1}{\bar{g}(\mathbf{0})} \int_{\mathbb{R}^{2}} K_{A_{1}^{-1}}\left(x_{1}, \omega_{1}\right) \mathscr{A}_{f, g}^{A_{1}, A_{2}}\left(\frac{\mathbf{x}}{2}, \boldsymbol{\omega}\right) \\
& \cdot K_{A_{2}^{-1}}\left(x_{2}, \omega_{2}\right) d \boldsymbol{\omega}
\end{aligned}
$$

provided that $\bar{g}(\mathbf{0}) \neq \mathbf{0}$.

In the same kind of reasoning as Theorem 13, one can easily derive Moyal's formula of the QAF-LCT as follows.

Theorem 22 (Moyal's formula for QAF-LCT). Let $f_{1}, f_{2}$, $g_{1}, g_{2} \in L^{2}\left(\mathbb{R}^{2} ; \mathbb{U}\right)$ be quaternion-valued signals. Then the following equation holds:

$$
\begin{aligned}
\operatorname{Sc} & \int_{\mathbb{R}^{2}} \int_{\mathbb{R}^{2}} \mathscr{A}_{f_{1}, g_{1}}^{A_{1}, A_{2}}(\mathbf{x}, \boldsymbol{\omega}) \overline{\mathscr{A}_{f_{2}, g_{2}}^{A_{1}, A_{2}}(\mathbf{x}, \boldsymbol{\omega})} d \boldsymbol{\omega} d \boldsymbol{\tau} \\
& =\left\langle f_{1}\left(\overline{g_{1}}, \overline{g_{2}}\right), f_{2}\right\rangle .
\end{aligned}
$$

\section{Conclusion}

In this paper, we have presented the quaternion WignerVille distribution associated with linear canonical transform (QWVD-LCT) and the quaternion ambiguity function associated with linear canonical transform (QAF-LCT). We provide alternative proof of well-known properties of the QWVD-LCT and QAF-LCT such as inversion formula and Moyal formula. The proof of the properties can be obtained using the properties of the QFT and relationship among the QWVD-LCT, QAF-LCT, and the QFT. We also studied that the QAF-LCT can be reduced to the QWFT and the CQWT.

\section{Conflicts of Interest}

The authors declare that there are no conflicts of interest regarding the publication of this paper.

\section{Acknowledgments}

This research is partially supported by WCU Percepatan Publikasi Internasional Tahun 2016 (no. 3834/UN4.21/PL.09/ 2016) from the Hasanuddin University, Indonesia.

\section{References}

[1] T. A. Ell, "Quaternion-Fourier transforms for analysis of twodimensional linear time-invariant partial differential systems," in Proceedings of the 32nd IEEE Conference on Decision and Control. Part 2 (of 4), pp. 1830-1841, December 1993.

[2] T. A. Ell and S. J. Sangwine, "Hypercomplex Fourier transforms of color images," IEEE Transactions on Image Processing, vol. 16, no. 1, pp. 22-35, 2007.
[3] T. Bülow, Hypercomplex Spectral Signal Representations for the Processing and Analysis of Images [Ph.D. thesis], University of Kiel, Germany, 1999.

[4] K. I. Kou, J.-Y. Ou, and J. Morais, "On uncertainty principle for quaternionic linear canonical transform," Abstract and Applied Analysis, vol. 2013, Article ID 725952, 2013.

[5] K. I. Kou and J. Morais, "Asymptotic behaviour of the quaternion linear canonical transform and the Bochner-Minlos theorem," Applied Mathematics and Computation, vol. 247, pp. 675688, 2014.

[6] Y. Yang and K. Ian Kou, "Uncertainty principles for hypercomplex signals in the linear canonical transform domains," Signal Processing, vol. 95, pp. 67-75, 2014.

[7] A. I. Zayed, "On the relationship between the Fourier and fractional Fourier transforms," IEEE Signal Processing Letters, vol. 3, no. 12, pp. 310-311, 1996.

[8] M. Bahri and R. Ashino, "A simplified proof of uncertainty principle for quaternion linear canonical transform," Abstract and Applied Analysis, Article ID 5874930, Art. ID 5874930, 11 pages, 2016.

[9] X.-L. Fan, K. I. Kou, and M.-S. Liu, "Quaternion Wigner-Ville distribution associated with the linear canonical transforms," Signal Processing, vol. 130, pp. 129-141, 2017.

[10] M. Bahri, "On two-dimensional quaternion Wigner-Ville distribution," Journal of Applied Mathematics, Article ID 139471, Art. ID 139471, 13 pages, 2014.

[11] L. Akila and R. Roopkumar, "Multidimensional quaternionic Gabor transforms," Advances in Applied Clifford Algebras, vol. 26, no. 3, pp. 985-1011, 2016.

[12] Y. Fu, U. Kähler, and P. Cerejeiras, "The Balian-low theorem for the windowed quaternionic Fourier transform," Advances in Applied Clifford Algebras, vol. 22, no. 4, pp. 1025-1040, 2012.

[13] S. Hartmann, "Some results on the lattice parameters of quaternionic Gabor frames," Advances in Applied Clifford Algebras, vol. 26, no. 1, pp. 137-149, 2016.

[14] S. Hartmann, "Relaxed quaternionic Gabor expansions at critical density," Mathematical Methods in the Applied Sciences, vol. 40, no. 5, pp. 1666-1678, 2017.

[15] M. Bahri, R. Ashino, and R. Vaillancourt, "Two-dimensional quaternion wavelet transform," Applied Mathematics and Computation, vol. 218, no. 1, pp. 10-21, 2011.

[16] M. Bahri and R. Ashino, "A variation on uncertainty principle and logarithmic uncertainty principle for continuous quaternion wavelet transforms," Abstract and Applied Analysis, Article ID 3795120, Art. ID 3795120, 11 pages, 2017.

[17] P. Fletcher and S. J. Sangwine, "The development of the quaternion wavelet transform," Signal Processing, vol. 136, pp. 2-15, 2017.

[18] R. Tao, Y. E. Song, Z. J. Wang, and Y. Wang, "Ambiguity function based on the linear canonical transform," IET Signal Processing, vol. 6, no. 6, pp. 568-576, 2012. 


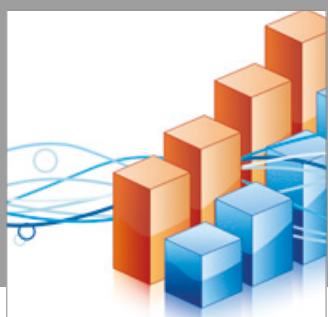

Advances in

Operations Research

vatersals

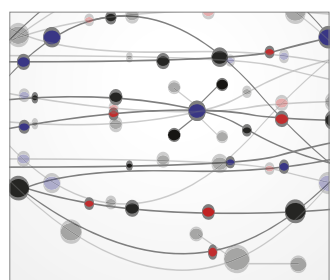

\section{The Scientific} World Journal
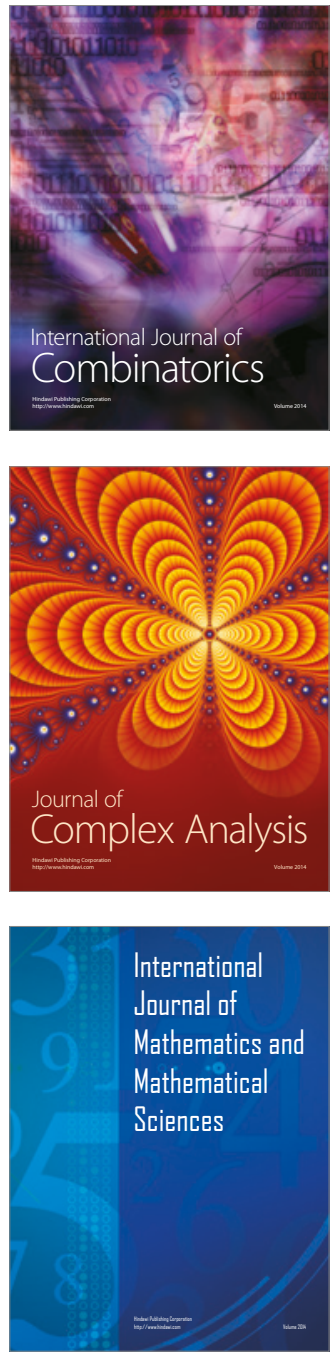
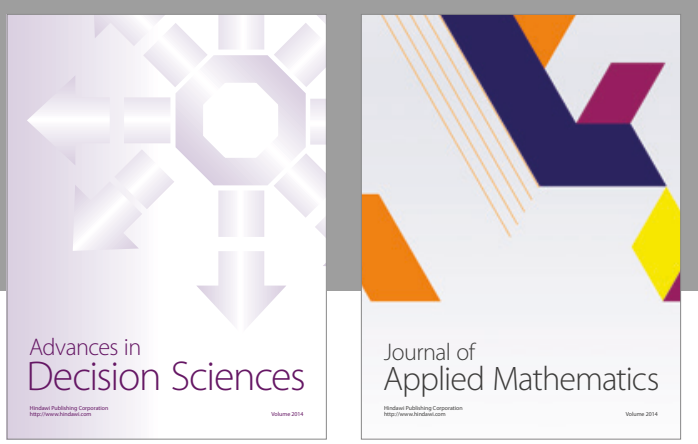

Algebra

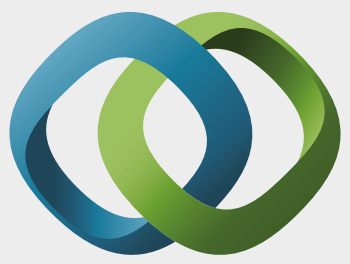

\section{Hindawi}

Submit your manuscripts at

https://www.hindawi.com
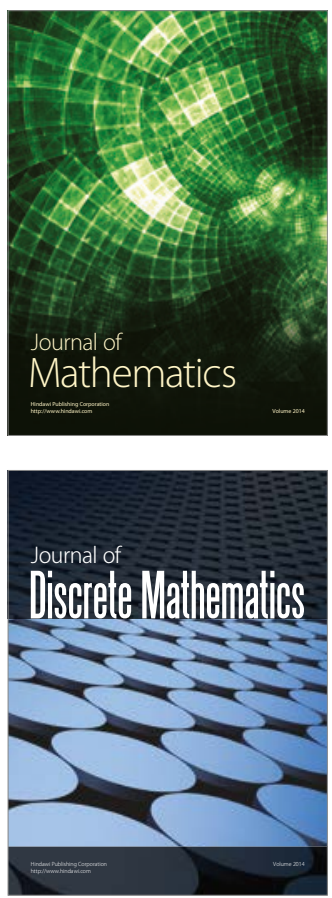

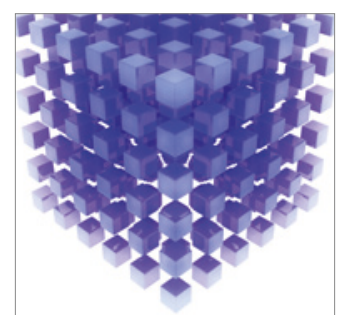

Mathematical Problems in Engineering
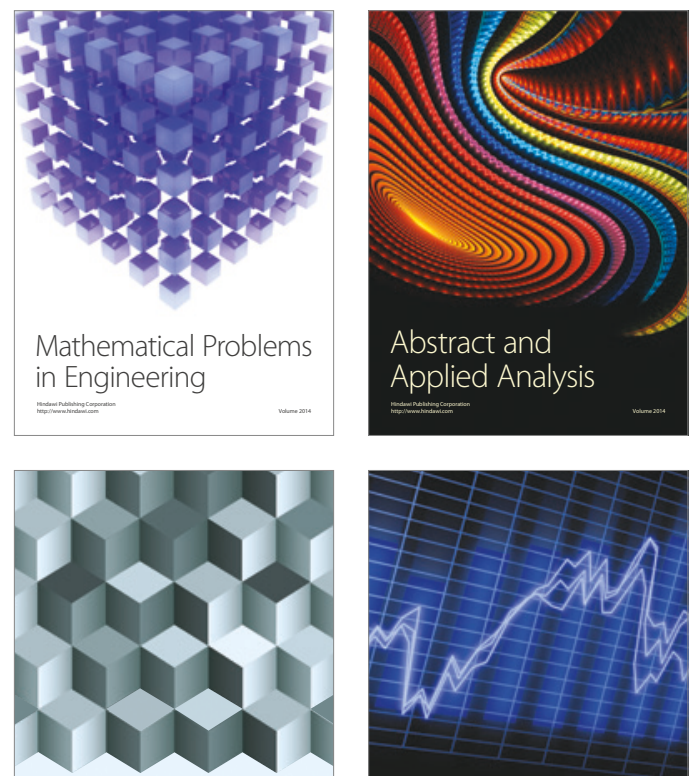

Journal of

Function Spaces

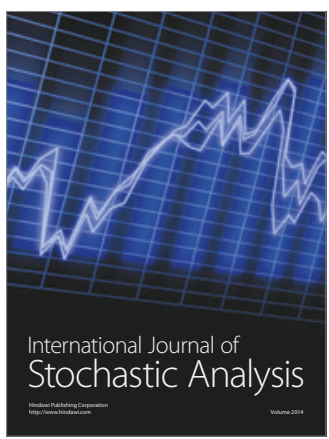

Probability and Statistics
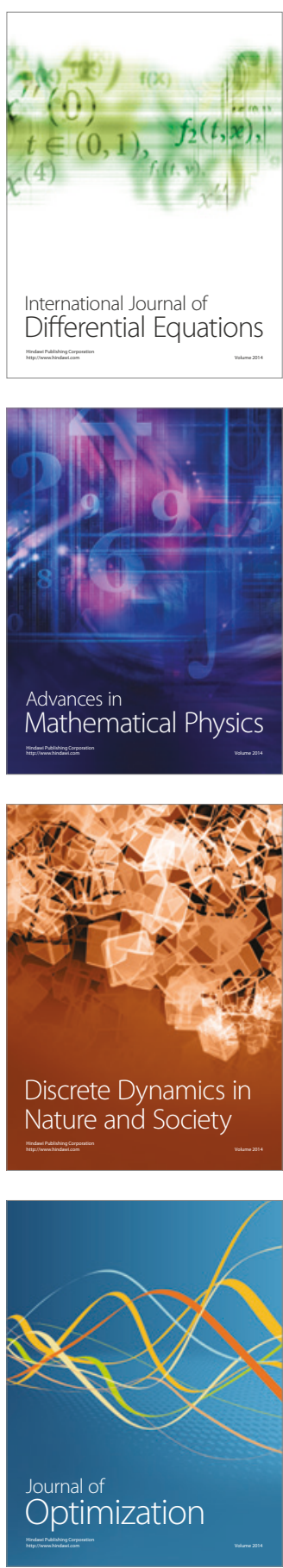